\title{
Motivação no serviço público: A relação entre a motivação e a qualidade do serviço público sob uma perspectiva geral
}

\author{
Hesler Piedade Caffé Filho ${ }^{\text {; Maria do Carmo Alves Gonçalves }{ }^{2} \text {; Naylane Leite dos Santos }}{ }^{3}$
}

Resumo: O presente artigo busca expor os principais fatores presentes nas teorias motivacionais alocando-os à realidade do serviço público, analisar quais elementos do ambiente estão ligados à atividade motivacional e ainda explorar o conteúdo acerca da cultura organizacional. A metodologia aplicada foi através de pesquisa descritiva, qualitativa e bibliográfica.

Palavras-chave: Motivação, organizações públicas, serviço público.

\section{Solid Waste in Santa Cruz da Venerada-PE: Disposal, Movement and Destination}

\begin{abstract}
The present article seeks to expose the main factors present in motivational theories by allocating them to the public service reality, analyzing which elements of the environment are linked to the motivational activity and still explore the content about the organizational culture. The applied methodology was through descriptive, qualitative and bibliographical research.
\end{abstract}

Keywords: Motivation, public organizations, public service.

\section{Introdução}

Nos dias atuais, a motivação do capital humano é relevante para tornar uma organização competitiva, pois os objetivos somente serão alcançados a partir do empenho das pessoas envolvidas neste processo. Acredita-se também que quando os colaboradores estão satisfeitos com o seu ambiente de trabalho certamente produzirão produtos e/ou serviços de excelência.

\footnotetext{
1 Administrador pela Estácio de Sá; MBA em Gestão Estratégica de Negócios pela Escola de Engenharia Eletromecânica da Bahia; Especialista em Marketing Institucional pela Faculdade São Francisco de Juazeiro (2009); Mestre em Gestão de Políticas Públicas da Universidade Federal do Recôncavo da Bahia. Administrador da Universidade Federal do Vale do São Francisco, lotado na Assessoria de Comunicação; Professor da Faculdade São Francisco de Juazeiro: Graduação em Administração e em Comunicação Social e Pós Graduação em Gestão Estratégica e em Marketing; Professor da Universidade Federal do Vale do São Francisco: Pós Graduação em Gestão Pública. Hesler.caffe@univasf.edu.br;

${ }^{2}$ Discente do curso de Pós-graduação (Lato Sensu) em Gestão Pública da Universidade Federal do Vale do São Francisco - UNIVASF.

E-mail: mcalvesgoncalves@ hotmail.com (Autor correspondente);

3 Administradora pela Universidade de Pernambuco (UPE), Discente do curso de Pós-graduação (Lato Sensu) em Gestão Pública da Universidade Federal do Vale do São Francisco - UNIVASF. E-MAIL: naylane00@ gmail.com (autor correspondente)
} 
Nesse sentido, torna-se necessário as organizações conhecerem os fatores motivacionais do seu colaborador para então buscar estratégias que estimulem o seu melhor desenvolvimento.

Como afirma Knapik (2006, p. 96) as organizações devem "incentivar as pessoas para a ação, para a realização e a conquista de objetivos, de modo a evitar a acomodação e a estagnação da criatividade e da inventividade".

É de suma importância que as pessoas se mantenham motivadas e que as relações interpessoais sejam harmônicas para auxiliarem a manutenção de um clima organizacional agradável. As relações humanas estão cada vez mais presentes no ambiente organizacional, então cobra-se da gestão um investimento cada vez maior na busca constante para mais envolvimento e comprometimento das pessoas com a organização. Como afirma Knapik (2006, p. 95) "as organizações são compostas de pessoas, que representam o coração da empresa, bombeando informações e ações para sua sobrevivência. Para que a relação homemempresa seja agradável e eficaz devemos buscar harmonia e equilíbrio de interesses."

Torna-se praticamente impossível uma organização alcançar seus objetivos quando há um relacionamento institucional incapaz de superar as divergências pessoais, discordâncias ou conflitos; é importante destacar que cada colaborador é uma peça fundamental na prospecção da imagem que o cliente/usuário terá da organização. Para aqueles que trabalham em contato direto com o público, a motivação pode ser um fator determinante para a qualidade de um serviço oferecido.

Para Marques (2012), uma organização eficiente procura entender as necessidades de seus clientes internos e externos e assume o compromisso com a qualidade, reforçando o relacionamento e interagindo com eles, a eficiência na prestação de seus serviços sempre será o objetivo principal. Sendo assim, o presente estudo buscará compreender a relação entre a motivação e a qualidade do serviço oferecido assim como sugerir ações de intervenção propostas para que os servidores possam ser estimulados a produzir com a qualidade devida e esperada.

O objetivo geral do presente estudo foi expor os principais fatores presentes nas teorias motivacionais alocando-os à realidade do serviço público. Para isso foi necessário: a) explorar o conteúdo das principais teorias motivacionais; b) analisar quais elementos do 
ambiente estão ligados à atividade motivacional; e, c) explorar o conteúdo acerca da cultura organizacional.

\section{Teorias Motivacionais}

Há muito tempo, busca-se encontrar explicações mais claras para a origem e definição do termo motivação; seja baseado em teorias elaboradas por filósofos ou estudiosos no assunto, ou mesmo a partir de experiências e observações no ambiente de trabalho, o fato é que a grande quantidade de conhecimento à disposição das organizações, privadas ou públicas, é uma ferramenta que quando bem colocada em prática traz benefícios capazes de melhorar o clima organizacional, o rendimento dos colaboradores e, consequentemente o aumento da produtividade.

Para Gondim e Silva (2004), de todos os aspectos envolvidos no conceito de motivação os principais e que geralmente repercutem na construção de teorias sobre esse processo psicológico são a ênfase, o foco, a pergunta e a resposta.

Para Gondim e Silva (2004), caso o destaque esteja na ativação, o principal questionamento recai sobre o que é capaz de desenvolvê-la, e a solução encontra-se em classificar os fatores intrínsecos e extrínsecos ao indivíduo. Escolhendo a direção, a indagação seria no sentido de se verificar a capacidade da pessoa dirigir e controlar o alvo, ou, de maneira diversa, se a direção está fora de seu controle consciente. Quando a ênfase for na intensidade, devido à ligação que esta possui com a variabilidade da força de ação que podem surgir a partir de necessidades a serem supridas ou objetivos para atingir, busca-se entender sua origem, se em virtude de um estado anterior de carência (necessidades) ou de um estado posterior a ser alcançado (objetivo). Enfim, a persistência da ação produz o questionamento sobre o que realmente a mantém, podendo ser por fatores subjetivos como necessidades, desejos, traços de personalidade e impulsos ou externos/físicos, como tipo de tarefa, equipe de trabalho, chefia, condições físicas, clima organizacional, recursos tecnológicos, salário, recompensas externas e reforço. Segundo eles, um arranjo destas quatro ênfases são a base para concepção das teorias motivacionais. 


\section{Teorias das Necessidades de Maslow}

O psicólogo Abraham. H. Maslow é um dos autores mais importantes quando se trata do estudo das teorias motivacionais voltadas para as necessidades humanas; a partir de observações, ele propôs que as necessidades ou motivos estão escalonados numa hierarquia.

Uma necessidade é uma condição qualquer que ocorre na pessoa e que é essencial ou necessária à sua vida, a seu crescimento e a seu bem estar (Reeve 2006) . A Teoria de Maslow primeiramente as classifica em cinco tipos e as dispõem na seguinte ordem de ascendência: fisiológicas, segurança, sociais, estima e auto-realização. Para Murray (1937 apud REEVE, 2006), todas as necessidades geram energia. O que difere uma necessidade da outra são seus efeitos direcionais sobre o comportamento.

Salgado (2005) menciona que as três primeiras são necessidades de carência, pois devem ser satisfeitas para que os indivíduos se sintam saudáveis e seguros e as duas últimas são as necessidades do crescimento, porque estão relacionadas ao desenvolvimento e à realização do potencial de cada pessoa.

Gondim e Silva (2004) observam que o desenvolvimento do homem somente ocorrerá com livre curso, se as necessidades inferiores (fisiológicas e de segurança) forem em parte satisfeitas e as superiores (sociais, estima e autorealização) apresentarem-se como motivadoras da conduta humana.

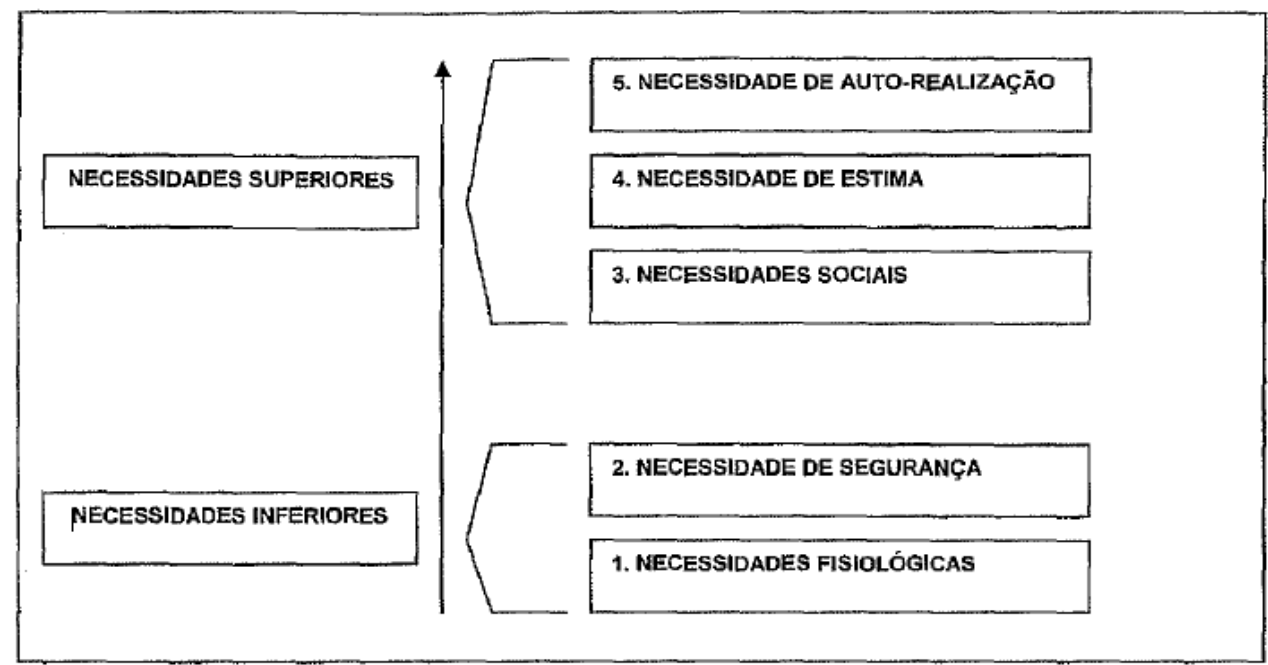

QUADRO 9: Hierarquia das Necessidades de Masiow Fonte: Gondim e Silva, 2004, p. 150 
Reeve (2006) afirma que juntas, as necessidades fisiológicas, psicológicas e sociais fornecem uma diversidade de motivos que serve de modo geral à nossa vida, ao nosso crescimento e ao nosso bem estar.

Hampton (2005) entende que a hierarquia das necessidades de Maslow é antes um reflexo da cultura onde surgiu do que um guia para se entender a motivação em outras culturas. Para ele, existem diferenças dentro de uma mesma cultura, as necessidades dos trabalhadores mudam ao longo dos tempos, o grau de intensidade da motivação, dentro das necessidades, varia de indivíduo para indivíduo e que a evidência de diferenças culturais e individuais nos padrões de necessidade sugere que os esforços para administrar motivação poderiam ser mais bem orientados por uma teoria modificada da hierarquia das necessidades.

\section{Teoria dos dois fatores de Herzberg}

A teoria da Motivação-Higiene trazida por Herzberg surgiu a partir de experimentos que questionavam os colaboradores sobre situações do trabalho nas quais eles se sentiam excepcionalmente bem e mal com relação aos seus cargos; segundo Salgado (2005) Herzberg concluiu que muitas das características do trabalho que estão associadas à insatisfação se apresentam bem diferentes daquelas associadas à satisfação; foi proposta então, a noção sobre a existência de dois fatores que influenciam na motivação para o trabalho, motivacionais ou intrínsecos e os higiênicos ou extrínsecos.

Gil (2001), afirma que os fatores higiênicos não são capazes de criar condições que motivem, entretanto, sua ausência gera insatisfação ou desequilibra o ambiente. Para Daft (2005), quando ruins, o trabalho se torna insatisfatório, entretanto, quando bons, apenas removem a insatisfação, pois eles, os fatores higiênicos, por si sós não induzem as pessoas a se tornarem altamente satisfeitas e motivadas em seu trabalho, posição que vai ao encontro da adotada por Chiavenato (2000) por entender que, quando ótimos, evitam a insatisfação e quando insuficientes, provocam insatisfação e, ainda, por Salgado (2005) que discorre:

Herzberg, que distinguiu, entre os fatores existentes, aqueles que ele considerava motivadores dos que ele considerava higiênicos, ou seja, aqueles cuja ausência ou desatenção implicava insatisfação dos trabalhadores, mas cuja presença, considerada 
satisfatória, não gerava nas pessoas qualquer satisfação pela realização do trabalho ou vontade de trabalhar (SALGADO, 2005, p. 4).

Os fatores higiênicos referem-se ao ambiente e possuem a função de impedir a insatisfação ao executar suas atividades, são as condições que cercam o indivíduo durante o trabalho, envolve fatores como ergonomia, salário, benefícios recebidos, supervisão, relacionamentos e contexto geral que envolve o cargo ocupado. Os fatores motivacionais, referem-se àquilo que é proporcionado para que cada pessoa consiga se desenvolver, através de conteúdo ou atividades desafiantes e estimulantes do cargo desempenhado, para que ela possa crescer profissionalmente e assumir maiores responsabilidades, sendo dessa maneira motivada em sua função através da realização de tarefas e deveres inerentes ao seu cargo.

Segundo Marras (2011) as organizações que possuem uma administração de recursos humanos mais tradicional se equivocam, na maioria das vezes, buscando satisfazer apenas os "fatores higiênicos", ou seja, criam programas que atendem necessidades de ordem primária dos indivíduos (restaurante, assistência médica, salários de mercado, etc) com isso, não ultrapassam o meio da pirâmide motivacional. O fato é que essas organizações ainda não entenderam que com essas atitudes não está "motivando" seu empregado, o máximo que ela conseguem com essas ações é satisfazer certas necessidades básicas de seus colaboradores. Satisfazer, portando, é diferente de motivar. O trabalhador poderá estar satisfeito, mas não necessariamente motivado.

\section{Teoria ERC}

Seguindo a mesma linha de raciocínio de Maslow, Alderfer (apud GONDIM e SILVA, 2004), no final da década de 1960, redefiniu as cinco necessidades hierarquizadas e as resumiu em três: existência, relacionamento e crescimento (ERC), relacionando a existência às necessidades presentes na base da pirâmide, fisiológicas e de segurança; o relacionamento, às sociais e de estima e o crescimento, à de auto-realização.

Para Daft (2005), o modelo ERC e a Hierarquia das Necessidades de Maslow são parecidos, devido à mesma disposição hierárquica e por presumirem que os indivíduos se movimentam para cima na hierarquia, um passo por vez, apesar de Alderfer ter reduzido a três 
as categorias de necessidades e proposto ser o direcionamento para cima na hierarquia mais complexo, refletindo um princípio de frustração-regressão. Nesse sentido, Daft (2005) observa

\begin{abstract}
Um funcionário que não consegue satisfazer uma necessidade para o crescimento pessoal poderá reverter para uma necessidade de ordem mais baixa e redirecionar seus esforços para poder fazer muito dinheiro. O modelo ERC, portanto, é menos rigoroso do que a hierarquia das necessidades de Maslow, sugerindo que os indivíduos podem se movimentar para baixo assim como para cima na hierarquia, dependendo da sua habilidade em satisfazer as necessidades (DAFT, 2005, p. 402).
\end{abstract}

\title{
Teoria das necessidades adquiridas (McClelland)
}

Na visão de David McClelland, o comportamento motivado pode ser estimulado por três necessidades; estas são consideradas secundárias, aprendidas ou adquiridas socialmente, denominadas realização, poder e afiliação. Essa teoria, conforme expressa Daft (2005), estabelece que determinados tipos de necessidades são adquiridos durante o tempo de vida do individuo, ou seja, as pessoas não nascem com essas necessidades, entretanto podem assimiláIas durante suas expectativas de vida.

A necessidade de realização é o desejo inconsciente de atingir um nível de excelência técnica ou profissional. Ser capaz de executar algo difícil com o domínio de atribuições complexas e o impulso de se fazer notar. Além disso, o interesse de realizar-se em relação a um conjunto de padrões; de ser bem-sucedido e de superar outras pessoas.

O poder envolve a necessidade de fazer com que outras pessoas ajam de uma maneira que, em outro contexto, se comportariam diferente, ou seja, o desejo de influenciar ou controlar outras pessoas, além de ser responsável pelos outros e de ter autoridade. É importante ressaltar que esta necessidade varia em intensidade de acordo com cada indivíduo. A afiliação compreende o desejo inconsciente de fazer parte de um grupo social acolhedor e é expressa pelo conjunto de impulsos que leva o individuo a procurar ou manter relações afetivas com outros indivíduos, a manutenção de relações interpessoais, por exemplo, a amizade, e o desejo de evitar conflitos.

Na compreensão de Hampton (2005) cada necessidade apresentada por McClelland está de alguma forma relacionada com àquelas discutidas por Maslow e por outros teóricos. 
Para ele, o poder, poderia ser comparado a um tipo particular de necessidade social ou de estima, destina-se a relações com pessoas e "status"; a afiliação pode ser tida como uma espécie de sinônimo para o que Maslow denominava de afeto; já a realização pode ser relacionada a alguns padrões de comportamento afiliados com a auto-estima ou a autorealização. O poder, a auto-realização e a afiliação são particularmente importantes visto que estão unidos a formas distintas de comportamento que podem aumentar ou reduzir as chances de sucesso no emprego.

Segundo Gondim e Silva (2004), McClelland embora admita que as necessidades surjam a partir de um processo biológico, ele não as considera segundo uma hierarquia; as três necessidades apresentadas em sua teoria se dispõem em níveis diferenciados de intensidade nas pessoas, de acordo com os seus perfis psicológicos e com os processos de socialização aos quais estiveram submetidos.A motivação para o trabalho dependerá dos arranjos entre esse três tipos de necessidades.

De acordo com Perez-Ramos e Robbins (1990 e 1998 apud GONDIM e SILVA, 2004), um dos méritos da teoria de McClelland foi o de ter destacado a importância do processo de socialização e da cultura na definição de perfis psicológicos mais ajustados ás demandas no ambiente de trabalho.

\section{Cultura Organizacional}

É notável na maioria das organizações integrantes do cenário atual a presença de uma diversidade cultural que pode ser compreendida sob as mais diversas óticas, sendo estas perspectivas positivas quando bem geridas e trazem bons resultados para empresa ou negativas, quando demasiadamente engessada tornando todo o sistema resistente à mudança. O conceito de Cultura Organizacional trazido por Chiavenato (2010) a inclui como um conjunto de hábitos estabelecidos por normas, crenças e atitudes compartilhadas por todos os membros da organização e que a partir de então contribui para distinguir aquela organização das demais, ou seja, é um conceito que está diretamente atrelado à sua identidade; assim como ocorre em cada indivíduo de forma singular, a sua cultura, comportamentos e reações às diversas situações transmitem sua individualidade perante os demais. 
Este constructo é amplamente defendido pela corrente idealista que traça a teoria da cultura de acordo com crenças, valores, expectativas e outros conceitos que influenciam as práticas de uma organização (REDMON E MASON, 2001). Shein (1996 apud VASCONCELOS-SILVA, TODOROV e SILVA 2012), também defensor daquela corrente, propõe que os sentimentos, valores e comportamentos que fazem parte das práticas culturais são determinados por percepções, linguagens e processos que um grupo passa a compartilhar.

Shein (1985) divide os níveis de cultura em três, sendo o primeiro os artefatos em que rituais, símbolos, estruturas e processos organizacionais visíveis são os mais importantes as serem observados; no segundo nível seriam os valores compartilhados onde as estratégias, metas e filosofia destacam-se e, por fim, as suposições básicas em que as crenças e sentimentos não externados representariam os dados a serem analisados; ele também afirma que para os grupos organizacionais existem dois principais desafios em lidar com os aspectos culturais a integração interna e a adaptação externa ambos os assuntos são tratados nos níveis comportamental, cognitivo e emocional; (PIRES E MACEDO, 2006) criticam essa abordagem pois, segundo eles, a deficiência consiste no fato de a análise não inserir a organização ou grupo estudado no contexto global. Hosfsedo (1994) igualmente crítica: "Todo ser humano é de fato o socializado de determinado meio, não se pode tornar inteligível a dinâmica humana nas organizações sem conhecer a cultura e a sociedade na qual ela se insere"

Dos elementos que compõem a cultura organizacional Chiavenato (2010) faz uma divisão entre os que são claramente perceptíveis e àqueles que não estão tão evidentes; os aspectos formais como políticas, diretrizes, métodos, procedimentos, objetivos, tecnologias entre outros que estão ligados aos processos formam o primeiro grupo chamado "ponta do iceberg" e os aspectos informais como percepções, sentimentos, atitudes, opiniões, relações afetivas são os aspectos ocultos que são mais difíceis de compreender, interpretar como também são mais resistentes às transformações. Este mesmo autor mostra a relação entre culturas mais flexíveis em organizações bem sucedidas mostrando a relação direta entre esses dois fatores, pois uma cultura aberta é capaz de acomodar as diferenças sociais e culturais dos seus colaboradores assim como as pessoas precisam integram e interagir entre as diferentes culturas organizacionais para serem também bem sucedidas. 
A presença de culturas conservadoras de adaptativas determina, em alguns casos, os rumos da organização em que estão inseridas. Culturas adaptativas são maleáveis e flexíveis e estão voltadas principalmente para inovação e mudança. O conservantismo mantém idéias, valores, costumes e tradições que permanecem arraigados e não mudam ao longo do tempo, as organizações conservadoras mantêm-se inalteradas como se nada houvesse no mundo ao seu redor sendo esta uma característica presente nas organizações públicas que pode ser considerada um entrave ao seu crescimento e desenvolvimento além das questões políticas que envolvem a sua gestão um fator a parte a ser analisado. (PIRES E MACEDO, 2006)

Martelane (1991) expõe que existem dois corpos funcionais distintos com características peculiares, um permanente e outro não permanente. Para ela, o corpo permanente é formado por trabalhadores de carreira, cujos objetivos e cultura foram formados no seio da organização e o não-permanente é formado por administradores políticos que seguem objetivos externos e mais amplos à organização. Schall (1997 APUD ) afirma que a descontinuidade administrativa causada pelo envolvimento político na gestão confere especificidades que podem ser claramente traduzidas para realidade brasileira como por exemplo: projetos de curto prazo, duplicação de projetos, conflitos de objetivos e administração amadora.

As organizações públicas de um modo geral, ou seja, em quaisquer ramos que possam vir atuar, possuem um alto grau de burocracia estatal, uma característica arraigada à sua cultura desde a formação inicial no século XIX. As condições e a organização do trabalho são padronizadas em todo setor público, logo, os servidores tendem a encontrar as mesmas condições laborais e de organização do trabalho proporcionadas pela burocracia estatal. É possível encontrar as mesmas características presentes nas demais organizações, porém acrescidas de algumas especificidades como apego às regras, supervalorização da hierarquia, paternalismo nas relações entre outros estes fatores são determinantes na definição dos processos internos, políticas de recursos humanos e formação dos valores e crenças. (PIRES E MACEDO, 2006) 


\section{Metodologia da Pesquisa}

Marconi \& Lakatos (2012) conceituam a pesquisa em si como um procedimento formal, de método reflexivo, que exige um tratamento científico e torna-se o principal caminho para conhecer a realidade ou descobrir verdades parciais. A partir da seleção de um questionamento inicial, ou problemática tem-se o ponto de partida para o desenvolvimento de um projeto de pesquisa, seguido da definição e diferenciação deste problema, levantamento de hipóteses de trabalho, coleta dos dados, análise e interpretação dos resultados e por fim a apresentação do relatório de pesquisa.

Como sempre parte de um tipo de problema a pesquisa obrigatoriamente deve responder às necessidades de conhecimento de determinado fenômeno; diante das várias hipóteses levantadas a pesquisa pode invalidá-las ou confirmá-las. A principal finalidade da pesquisa é descobrir respostas para questões mediante a aplicação de métodos científicos (MARCONI E LAKATOS 2012).

A pesquisa em contexto se classifica, quanto aos objetivos, como descritiva que segundo Vergara (2009), tem o objetivo descrever características de determinada população ou determinado fenômeno assim como pode estabelecer correlações entre variáveis e definir sua natureza. "Não tem o compromisso de explicar os fenômenos que descreve, embora sirva de base para tal explicação.” Vergara (2009). Para (OLIVEIRA 2011) o estudo descritivo deve demonstrar exatamente a realidade de modo que seja utilizado quando a intenção do pesquisador é conhecer determinada comunidade, suas características, valores e problemas relacionados à sua cultura. Segundo Gil (1999) a pesquisa descritiva pode abranger o estudo do nível de atendimento nos órgãos públicos com o objetivo de levantar opiniões, atitudes e crenças de uma população.

Quando se diz que uma pesquisa é descritiva, se está querendo dizer que se limita a uma descrição pura e simples de cada uma das variáveis,isoladamente, sem que sua associação ou interação com as demais sejam examinadas (CASTRO, 1976, p. 66).

Quanto à sua natureza podemos classificá-la como qualitativa que segundo Bogdan \& Biklen (2003 apud OLIVEIRA 2011) envolve a obtenção de dados descritivos resultantes do contato direto do pesquisador com a situação estudada, onde a ênfase maior concentra-se mais 
no processo do que no produto em si. Estes autores enfatizam que a pesquisa qualitativa supõe uma vivência intensa entre o pesquisador e a situação que está sendo investigada por meio do trabalho intensivo de campo.

O significado que as pessoas pesquisadas dão às coisas e à sua vida é foco de atenção especial do pesquisador onde é destaque a intenção de capturar a "perspectivas dos participantes", ou seja, como eles encaram os questionamentos que lhes são oferecidos. (OLIVEIRA, 2011)

Quanto à técnica de coleta de dados tem-se que a pesquisa bibliográfica é a que mais se enquadra para tal contexto, pois trata-se de uma busca em fontes secundárias provenientes de outras pesquisas realizadas e publicadas em livros, jornais, periódicos, teses, materiais cartográficos, enfim, nas mais diversas literaturas. A pesquisa bibliográfica oferece a possibilidade de fornecer o instrumental analítico para qualquer outro tipo de pesquisa como também pode esgotar-se em sim mesma (OLIVEIRA, 2013).

\section{Proposta de Intervenção}

Observa-se atualmente entre as organizações públicas uma grande demanda quantitativa e qualitativa advinda dos cidadãos usuários que reflete diretamente para o servidor/prestador dos serviços tendo em vista o dever de eficiência previsto na Constituição Federal que é definida por Alexandrino e Paulo (2012) como uma noção de administração gerencial onde existe a intervenção mínima do Estado e a atuação da Administração seria semelhante à das empresas do setor privado onde há a ênfase no atingimento de resultados e a tentativa de reduzir os controles das atividades-meio; ainda há destaque na definição trazida por estes autores para excelência esperada no desempenho das atribuições do cargo e produtividade equiparável à que se verifica entre os melhores trabalhadores da iniciativa privada.

Nesta conjectura é possível identificar uma disparidade entre os deveres do servidor e o que a ele é oferecido por parte da administração pública; entre os gestores torna-se um grande desafio a obrigação de cobrar um nível de resultado se, em contrapartida, não seria possível oferecer condições estruturais para este ser alcançado. A valorização profissional 
seria um ponto inicial a ser trabalhado entre as entidades formadoras do setor público para Knapik (2011) a valorização de pessoa é uma questão estratégica e está atrelada aos processos de motivação, retenção e desenvolvimento de talentos. Ações que busquem a valorização profissional do servidor principalmente aquelas voltadas para suas atividades no cargo sugerem que haja maior retenção do profissional no intuito de evitar a rotatividade não só pela estabilidade garantida legalmente, mas também por despertar interesse no que efetivamente é oferecido por sua função pública.

Conforme foi tratado pela literatura e já citado acima a tendência da administração pública neoliberalista é comportar-se semelhantemente às empresas privadas e nestas um bom programa de remuneração e recompensas alavanca um comprometimento já que as recompensas aliadas aos desafios tentem a fazer o empregado vestir a camisa da empresa e comprometer-se com os resultado (KANPIK, 2011), temos então uma troca indireta entre instituições e seus funcionários estabelecida através da ferramenta motivadora remuneratória que supre as necessidades higiênicas extrínsecas dos seres. Em quaisquer situação espera-se que um aumento na remuneração gere um aumento na parcela de comprometimento com as atividade e as metas, porém no setor público este tipo de incentivo deve ser usado com cautela pois a demanda não é somente quantitativa, mas também qualitativa para implementação deste tipo de motivador seria necessário uma ferramenta de controle e mensuração de resultados já que não é permitido legalmente a supressão de quaisquer parcela remuneratória podendo então gerar uma situação de comodismo.

A capacitação e a conscientização dos indivíduos propiciam as condições mínimas para que haja uma gestão saudável no ambiente de trabalho e consequentemente um aumento nos níveis de produtividade, qualidade dos produtos e serviços e redução de custos (KNAPIK, 2011). Oferecer um ambiente de trabalho adequado para a realização das atividades é capaz de gerar um aumento na produtividade como também produz um impacto psicológico positivo para o funcionário. A salubridade do ambiente aliada à um programa de apoio e desenvolvimento de atividades que visem à prevenção ao stress, ansiedade e outras doenças psicossomáticas é um investimento válido, pois para Kanpik (2011) as doenças funcionais causadas pelo stress têm evidenciado um quadro preocupante sendo a qualidade de vida no trabalho um fator diferencial que assegura uma conscientização para organização do trabalho, bem estar dos colaboradores e aumento da produtividade. Segundo Chiavenato (2009) para 
atender e satisfazer o cliente externo, as organizações precisam cuidar antes de seus funcionários, responsáveis pelo produto ou serviço oferecido, a otimização do potencial humano é peça fundamental para o sucesso a qualidade de vida no trabalho (QVT) está assumindo importância sem precedentes no sucesso organizacional ela depende do grau de satisfação das pessoas em relação à empresa e ao ambiente de trabalho.

\section{Conclusão}

O artigo trouxe, inicialmente, como objetivo geral a explanação sobre as principais teorias acerca de motivação, exploradas no referencial teórico através de autores que sinteticamente abordam em suas obras estas teorias que são elas: Teoria das Necessidades de Maslow, Teorias dos Dois Fatores de Hezrberg, Teoria ERC e Teorias das Necessidades Adquiridas (McClelland) e ainda uma breve explanação acerca do tema Cultura Organizacional.

Através dessa pesquisa bibliográfica foi possível mensurar a importância de traçarmos um paralelo entre as definições literárias acerca do tema motivação e de qual forma ele apresenta-se no cenário das organizações públicas como também poderia ser realizado este estudo para que, na prática, cause um efeito positivo diante das peculiaridades presentes nessas organizações. Foi possível sugerir práticas a serem implantadas por parte dos gestores, estes, possuem um enorme desafio que é adaptar as diversas situações características da iniciativa privada para a causa pública.

Manter um servidor motivado e ser capaz de identificar quais tipos de fatores são capazes de causar efeito na sua produtividade requer um detalhamento minuncioso através de uma pesquisa específica que explore caso a caso.

Conhecer a realidade do serviço público no país, que por muito é tido como caótico e sem qualidade, sob outra perspectiva: a administrativa e teórica trata-se de uma experiência desafiadora. Principalmente por discorrer de forma tão específica e ser possível por fim, extrair metodologias de gestão capazes de causar um impacto positivo na vida profissional dos servidores como também um potencial e inevitável aumento na qualidade do serviço prestado. 


\section{Referências}

ALEXANDRINO, M. e PAUlO, V. Direito Constitucional Descomplicado. 9. Ed. São Paulo: MÉTODO, 2012.

BOGDAN, R. S.; BIKEN, S. Investigação qualitativa em educação: uma introdução à teoria e aos métodos. 12.ed. Porto: Porto, 2003.

CASTRO, C. M. Estrutura e apresentação de publicações científicas. São Paulo: McGraw-Hill, 1976.

CHIAVENATO, I. Recursos Humanos - São Paulo: Atlas, 2000.

CHIAVENATO, I. Recursos Humanos: o capital humano das organizações. 9.ed. - Rio de Janeiro: Elsevier, 2009.

DAFT, R. L. Administração. São Paulo: Thomson, 2005.

GIL, A. C. Métodos e técnicas de pesquisa social. São Paulo: Atlas, 1999.

GIL, A. C. Gestão de Pessoas: Enfoque nos Papéis Profissionais. São Paulo:

Atlas, 2001.

GONDIM, S. M. Gs; SILVA, N. Psicologia, Organizações e Trabalho no Brasil. Porto Alegre: Artmed, 2004.

HAMPTON, D. R. Administração Contemporânea. São Paulo: Pearson Makron Books, 2005.

KNAPIK, J. Gestão de Pessoas e talentos. 3. Ed. rev., atual. e ampl. - Curitiba: Ibpex 2011.

MARCONI, M. A; LAKATOS, E.M Técnicas de Pesquisa: planejamento e execução de pesquisas, amostragens e técnicas de pesquisa, elaboração, análise e interpretação de dados. 7. Ed. São Paulo: Atlas 2012.

MARRAS, J. P. Administração de Recursos Humanos do Operacional ao Estratégico. São Paulo, Saraiva, 2011.

MARTELANE, R. O relacionamento entre os corpos permanentes e não-permanentes na organização pública - um modelo. In: REUNIÃO ANUAL DA ANPAD, 15., 1991, Salvador, BA, Anais... Salvador: Anpad, 1991.

OLIVEIRA, M. F. Metodologia Científica: um manual para realização de pesquisas em administração. Catalão, UFG, 2011.

PIRES, J. C. de S.; MACEDO, K. B. Cultura organizacional em organizações públicas no Brasil. Rev. Adm. Pública, Rio de Janeiro , v. 40,n. 1, p. 81-104, Feb. 2006 . Available from<http://www.scielo.br/scielo.php?script=sci_arttext\&pid=S0034- 
$76122006000100005 \& \operatorname{lng}=\mathrm{en} \& \mathrm{nrm}=\mathrm{iso}>$. on 17 May 2017. http://dx.doi.org/10.1590/S0034-76122006000100005.

REDMON, W. K. \& MASON, M. A. Organizational culture and behavioral systems analysis. C. M. Johnson, W. K. Redmon \& T. C. Mawhinney (Eds.) Handbook of organizational performance: behavior analysis and management. (pp. 437-456). New York: The Haworth Press, 2001.

REEVE, J. Motivação e Emoção. Rio de Janeiro: LTC, 2006.

SALGADO, L. Motivação no Trabalho. Rio de Janeiro: Qualitymark, 2005.

SCHEIN, E. H. Organizational culture. American Psychologist, 45,109-119, 1990.

SCHEIN, E. H. Three cultures of management: the key to organizational learning. Sloan Management Review, 38(1), pp. 9-20, 1996.

VERGARA, S. C. Projetos e relatórios de pesquisa em administração. $8^{a}$ ed.São Paulo: Atlas, 2009.

VASCONCELOS-SILVA, A; TODOROV, J. C.; SILVA, R. L. F. C. Cultura organizacional: a visão da análise do comportamento. Rev. bras. ter. comport. cogn., São Paulo , v. 14, n. 2, p. 4863, ago. 2012. Disponível

em <http://pepsic.bvsalud.org/scielo.php?script=sci_arttext\&pid=S151755452012000200005\&lng=pt\&nr $\mathrm{m}=\mathrm{iso}>$. Acesso em 02 maio 2017.

\section{Como citar este artigo (Formato ABNT):}

CAFFÉ FILHO, Hesler P.; GONÇALVES, Maria do Carmo A.; SANTOS, Naylane L. Motivação no serviço público: A relação entre a motivação e a qualidade do serviço público sob uma perspectiva geral. Id on Line Revista Multidisciplinar e de Psicologia, Julho de 2017, vol.11, n.36, p.268-283. ISSN: 1981-1179.

Recebido: 03.07.2017

Aceito: 07.07.2017 